Anno 35, 2020 / Fascicolo 2 / p. 10-24 - www.rivista-incontri.nl - http://doi.org/10.18352/incontri. 10349 (C) The author(s) - Content is licensed under a Creative Commons Attribution 3.0 Unported License Publisher: Werkgroep Italië Studies, supported by Utrecht University Library Open Access Journals

\title{
Deus ex machina Forme contemporanee del credere nel relativo
}

\author{
Enzo Pace
}

La letteratura è il discorso teorico dei processi storici. Michel de Certeau ${ }^{1}$

\section{Introduzione}

Nel teatro greco, soprattutto nelle opere di Euripide, l'apparizione in scena del deus ex machina (apó machinés théos) serviva a risolvere una trama drammaturgica divenuta intricata. Poi, la formula è diventata un'espressione passe-partout per indicare genericamente l'intervento di una persona dotata di carisma che, grazie ai suoi poteri personali, riesce a risolvere un problema altrimenti irrisolvibile.

Vorrei usare questa figura del teatro greco per analizzare alcune tendenze che oggi rendono vivace (a volte furiosamente vivace) il panorama religioso contemporaneo. Ne farò un uso fantasioso, dilatando arbitrariamente i significati dell'artificio scenografico greco. In particolare, mi servirò di questa figura per fare il punto sul dibattito (ormai stucchevole, dal momento che perdura da troppo tempo, almeno dai primi anni Ottanta del secolo scorso) fra i sostenitori del paradigma teorico della secolarizzazione e i fautori dell'ipotesi del ritorno degli dei, fra quanti pensano che la fase storica segnata dai processi di secolarizzazione si sia compiuta per entrare in una nuova età: caratterizzata dalla de-secolarizzazione, per alcuni, per altri dal post-secolare, e per altri ancora dalla rinnovata affermazione delle religioni pubbliche (bene generale di una comunità politica, tutelato da uno Stato, anche quando questo si proclama formalmente non confessionale o laico).

In tale prospettiva, mi sembra necessario tornare ai classici della sociologia per parlare della machinés socio-economica chiamata capitalismo; di come la 'gabbia d'acciaio' di cui parlava Weber e 'il capitalismo come religione' di cui parlava Walter Benjamin si siano appropriati dei simboli religiosi, trasformando gradualmente gli habitus etici che originariamente si erano nutriti dei modelli ascetici definiti dal puritanesimo calvinista, in beni di consumo e in stili di vita conformi alla moda e al branding. La tesi che intendo sostenere è che il capitalismo neoliberista fa della religione dei consumi una delle sue forze motrici, per espandersi a livello mondiale, valicando frontiere culturali, linguistiche e religiose.

In particolare, tale esito non riguarda solo il dominio crescente del capitale finanziario su quello produttivo, già previsto da Karl Marx, ma anche e soprattutto il fenomeno che, per brevità, chiamerò di trasformazione del religioso in un brand: in un repertorio di segni e simboli, tratti dalle memorie di lunga durata delle grandi

\footnotetext{
1 'Le roman psychanalytique', in: M. De Certeau, Histoire et psychanalyse entre science et fiction, Paris, Gallimard, 1988, p. 125.
} 
religioni mondiali, riutilizzati per altri scopi e in altre province di significato. Il bene "religione" entra nel mercato mondiale e, in tal modo, su di esso settori produttivi importanti (da quello alimentare al biologico, dai cosmetici al vestiario) investono per mettere in circolazione merci che si presentino come conformi ai precetti di questa 0 quell'altra fede. Tramite le merci un credente può incorporare il segno di appartenenza a una ipotetica comunità di fede. Siamo di fronte a un'economia politica dei segni religiosi, per parafrasare un noto saggio di Baudrillard. ${ }^{2}$ Un campo di studi sempre più promettente, dove si sono accumulate conoscenze e ricerche sul tema, muovendo da ambiti disciplinari diversi: dalla semiotica alla sociologia, dagli studi sui nuovi media interessati alla digital religion alla scienza politica fino a quella economica. Sotto le sacre volte delle religioni, la machina neocapitalista può aiutare a rafforzare le politiche d'identità che leader e movimenti neo-nazionalisti da tempo cercano di promuovere. ${ }^{3}$

La religione può diventare un marcatore d'identità collettiva. La religione, infatti, produce simboli collettivi (a volte per grandi masse di persone) che possono essere incorporati e, in alcuni casi, portati addosso come abiti che distinguono dagli altri. Quando ciò accade, un bene simbolico può essere manipolato e diventare merce. In tutti e due i casi siamo di fronte a un effetto, forse più duraturo della secolarizzazione: credere diventa una scelta soggettiva, uno stile di vita individualizzato ${ }^{4}$ che mette in crisi sia il credere per tradizione sia l'appartenenza a comunità di fede radicate in un determinato territorio. Tuttavia, tale modo di vivere il rapporto con la religione è divenuto anch'esso ostaggio del mercato, che non solo tramite la comunicazione pubblicitaria, ma anche direttamente nei prodotti di consumo, rende a portata di mano (e di tasca) la trascendenza. Gli stili di consumo si orientano verso quelle merci che portano i segni della religione. Dal lato dell'offerta si moltiplicano, di conseguenza, soprattutto tramite i nuovi media, i messaggi per convincere le persone, ad esempio, che quel cibo è buono da mangiare perché rispetta la legge religiosa e la memoria che di essa ha tutta una comunità di fedeli.

Da un lato, dunque, la secolarizzazione continua a produrre i suoi effetti nella lunga durata, dall'altro, la moderna società dei consumi salva il salvabile, incorporando nella merce il sacro, facendo diventare le merci un segno di identificazione, un cult. L'apparizione di religioni fort $i,{ }^{5}$ i così detti fondamentalismi, ${ }^{6}$ costituisce una risposta speculare rispetto alle forme del credere nel relativo, che si affermano nelle società della tarda secolarizzazione e che hanno conosciuto già, nel respiro lungo della storia, i processi di disincanto e di individualizzazione delle credenze religiose.

\section{Eclissi del sacro o ritorno degli dei?}

L'immagine del deus ex machina mi è spesso venuta in mente quando, a partire dalle seconda metà degli anni Ottanta (del secolo appena trascorso), fra i sociologi della religione si è fatta strada l'idea che il paradigma della secolarizzazione fosse stato sconfessato ampiamente da un insieme di fenomeni che erano raggruppati sotto la formula del 'ritorno di Dio' o della 'rivincita di Dio'. ${ }^{7}$ Se in un primo match Dio era stato sconfitto, stavamo allora assistendo a una partita di ritorno, in cui Dio si

\footnotetext{
2 J. Baudrillard, Pour une critique de l'économie politique du signe, Paris, Gallimard, 1995 (trad. it. Milano, Mimesis, 2012).

${ }^{3}$ E. Pace, Perché le religioni scendono in guerra, Roma-Bari, Laterza, 2003.

${ }^{4}$ Su questo tema L. Berzano, La quarta secolarizzazione, Milano, Mimesis, 2017.

${ }^{5}$ Si tratta di una felice formula usata da G.A. Almond, S. Appleby \& E. Sivan, Strong Religion, Chicago, Chicago University Press, 2005 (trad.it. Religioni forti, Bologna, il Mulino, 2006).

${ }^{6}$ E. Pace \& R. Guolo, I fondamentalismi, Roma-Bari, Laterza, 2000.

7 Si tratta del titolo di un fortunato libro di G. Kepel, La revanche de Dieu, Paris, Seuil, 1991 (trad. it. Milano, Rizzoli, 1991).
} 
riprendeva la sua rivincita. Scendeva di nuovo in campo e aveva la meglio, a dispetto di tutti i punti segnati in epoca moderna dalla "squadra" avversaria.

All'idea della religione che perdeva di peso sociale, non potendo più influenzare e controllare decisive sfere della vita collettiva e dei mondi vitali (dalla politica all'economia, dal diritto alla formazione della conoscenza scientifica, dall'arte all'eros) si contrapponeva l'evidenza di un ritorno della religione nello spazio pubblico, nelle lotte politiche, nei processi di cambiamento storico: dalla prevista privatizzazione della religione ${ }^{8}$ alle religioni pubbliche, testimoniate sin dagli inizi degli anni Ottanta del secolo scorso da avvenimenti decisivi quali la rivoluzione iraniana del 1978-79 e quella polacca, non violenta e condotta contro un regime di stampo sovietico da Solidarność. Qui ebbero un ruolo di primo piano i cattolici, guidati da un Papa carismatico come Karol Woytita, ${ }^{9}$ mentre a Teheran erano i musulmani sciiti che mostravano al mondo intero come fosse possibile instaurare un regime teologicopolitico della verità sotto le forme repubblicane. Dio sembrava ridisceso in terra, provava a sciogliere i nodi irrisolti della storia contemporanea, la stessa che ne aveva decretato la morte: l'autonomia della politica e dell'economia dalla religione.

Da allora a oggi la discussione continua: fra chi sostiene la validità del paradigma della secolarizzazione e chi lo contrasta e invita a riflettere non solo sulla persistenza del sacro e del religioso, ma anche sulla sua vitale ripresa. ${ }^{10}$

I teorici della secolarizzazione più avveduti hanno cercato di argomentare a favore del progressivo declino del sacro e del religioso nelle società moderna distinguendo tre livelli. Da quello macro-sociale (dove è più evidente la progressiva autonomizzazione delle sfere sociali, la differenziazione in tanti sub-sistemi ciascuno dei quali, avrebbe detto Luhmann ${ }^{11}$ si organizza in base a un proprio codice interno diverso da quello trascendenza/immanenza che caratterizza ciò che chiamiamo religione) a quello meso-sociale (dove gli individui indossano i vari habitus imposti dai differenti ruoli predefiniti dai vari sub-sistemi di cui fanno parte come cittadini, lavoratori, professionisti, sportivi, e così via), a quello, infine, micro-sociale (dove un individuo può coltivare lo spirito, continuare a credere, praticare, affiliarsi o meno e così via, senza che tutto ciò incida nel gioco sociale o sia riconosciuto da parte dello Stato)..$^{12}$

A immagine e somiglianza di questa teoria a tre stadi, i sostenitori del ritorno di Dio o degli dei vedono invece una progressiva marcia di riavvicinamento che, muovendo dal micro arriva al macro. Dalla religione che avrebbe dovuto ridursi a un fatto privato (l'uomo nuovo moderno, sia quello concepito dal positivismo sia quello di matrice marxista-sovietica) e alla fine salire in cielo senza far più ritorno in terra, si passa a una fase storica, quella presente, in cui la religione torna a pretendere di dire la sua sull'ordine sociale e sulle forme politiche che governano le società umane.

Siamo di fronte a una discussione interna al campo della sociologia e di altre e più gloriose scienze umane (la filosofia e la scienza politica) che, in realtà, si condanna a un'infinita contrapposizione condotta, almeno in campo sociologico, con un dispendio di energie (umane e finanziarie) per accumulare dati di ricerca trattati con complesse procedure statistiche che, a ben vedere, non fanno altro che confermare

\footnotetext{
8 Sulla privatizzazione della religione cfr. T. Luckmann, The Invisible Religion, London, McMillan, 1967 (trad. it. Bologna, Il Mulino, 1968), mentre sul ritorno nella sfera pubblica delle religioni J. Casanova, Public Religion in the Modern World, Chicago, Chicago University Press, 1994 (trad. it. Oltre la secolarizzazione, Bologna, il Mulino, 2000).

${ }^{9}$ P. Michel, La société retrouvée, Paris, Fayard, 1988.

10 Per un bilancio critico delle teorie contrapposte L. Diotallevi, Fine corsa, Bologna, EDB, 2018.

11 Cfr. N. Luhmann, Funktion der Religion, Frankfurt a. M., Suhrkamp, 1977 (trad.it. Funzione della religione, Brescia, Morcelliana, 1991).

12 Su questa differenziazione dei tre livelli della secolarizzazione rinvio al fondamentale saggio di $\mathrm{K}$. Dobbelaere, Secularization: An Analysis at Three Levels, Bruxelles, Peter Lang, 2002.
} 
gli elementi del puzzle difronte a cui ci troviamo. ${ }^{13}$ L'immaginazione sociologica, del resto, paga un prezzo all'origine stessa della disciplina. Comte ${ }^{14}$ pensava, infatti, che il progresso scientifico avrebbe svuotato di senso la religione, trasferendo nella razionalità della scienza moderna (dalle scienze naturali alla fisica sociale, come chiamava all'inizio ciò che poi ribattezzerà sociologia) alcuni principi costitutivi del pensare religioso (l'unità al di là del molteplice, la connessione causa-effetto fra i fenomeni e così via). L'ultimo Comte, che immagina di poter sostituire alle religioni della trascendenza una nuova religione dell'umanità, si dovrà accontentare di veder sorgere in Europa e nel lontano Brasile delle chiese spiritiste razionaliste, che certo non sono divenute di massa.

Per uscire dal dilemma, bisogna porsi una domanda molto semplice: di cosa stiamo parlando, quando parliamo di religione nella società contemporanea? $\mathrm{Ci}$ aiuta poi, per rispondere alla domanda, tenere assieme sacro e religione? Il primo non si esaurisce nella seconda, dal momento che, dal punto di vista storico, ciò che chiamiamo religione è un sistema di credenze e pratiche, di concezioni astratte e di riti concreti, di passioni/emozioni individuali spesso regolati da un ceto di specialisti di sapere e potere religiosi, ${ }^{15}$ chiamato ad amministrare un sacro che non si esaurisce nel religioso. Perciò, il sacro forse non se n'è andato mai via dalla storia, mentre il religioso può conoscere alterne fortune nel corso della storia umana. Per cui, nella diatriba fra i teorici della secolarizzazione come processo inevitabile e i sostenitori del ritorno (dunque, di teorici della secolarizzazione pentiti), si fanno largo quanti indicano una terza via: il post-secolare. Si tratta di una formula che vanta tra i suoi sostenitori robusti pensatori contemporanei tra cui Jürgen Habermas. ${ }^{16}$

Di cosa si tratta, quando si parla di post-secolare? Nel mutato scenario del mondo occidentale, dopo la fine della guerra fredda (Berlino è il luogo dove fisicamente tale evento si è materializzato, polverizzando nello spazio di una notte di novembre del 1989 il muro che divideva in due la città tedesca), il protagonismo pubblico di attori identificabili come religiosi (movimenti di lotta, Chiese, sette, monaci usciti dalle celle, telepredicatori e nuovi leader carismatici capaci di creare nuove religioni) mette in discussione la forma moderna dello Stato non confessionale, laicamente neutro nei confronti delle credenze e pratiche religiose presenti nella società. Lo Stato non può non prestare attenzione a ciò che tali attori hanno da dire pubblicamente, riconoscendo loro il diritto (di cittadinanza) a concorrere nel definire le basi etiche comuni del patto sociale. Tutto ciò non significa però riconoscere la pretesa da parte di un attore religioso a imporre il proprio punto di vista in società aperte e plurali, abitate dalla diversità religiosa; in alcuni casi in Europa: da una super-diversità religiosa. ${ }^{17}$

Riassumendo per grandi linee la teoria del post-secolare, si può notare come essa costituisca un paradigma analitico che si colloca in mezzo al guado: da un lato i sostenitori della secolarizzazione come processo irreversibile di fine della religione

\footnotetext{
13 Sul puzzle della secolarizzazione applicato all'Italia si veda L. Diotallevi, Il rompicapo della secolarizzazione italiana, Soveria Mannelli, Rubbettino, 2001.

${ }_{14} \mathrm{~A}$. Comte, Cours de philosophie positive, Paris, Rouen Frères Ed., 1830.

15 Nella formulazione classica che ne dà M. Weber, Economia e Società, Milano, Edizioni di Comunità, 1961, vol. II, pp. 105 e segg. Tale nozione è stata ripresa e sviluppata dalla teoria del campo religioso da $\mathrm{P}$. Bourdieu, 'Genèse et structure du champ religieuX', in: Revue Française de Sociologie, XII (1971), pp. 295-334.

${ }^{16} \mathrm{Di}$ questo autore si veda soprattutto 'Notes on Post-Secular Society', in: New Perspective Quaterly, 25, 4 (2008), pp. 17-29. Inoltre, sul tema rinvio a C. Taylor, Secular Age, Harvard, Harvard University Press, 2007 (trad. it. L'età secolare, Milano, Feltrinelli, 2009). Per una ricostruzione puntuale del dibattito sul post-secolare vedi P. Costa, La città post-secolare, Brescia, Queriniana, 2019.

17 Sulla nozione di super-diversità etnica e religiosa si veda S. Vertovec, 'Super-diversity and its Implications', in: Ethnic and Racial Studies, 30, 6 (2007), pp. 1024-1054.
} 
nella modernità, dall'altro coloro che vedono dappertutto il ritorno degli dei. Infatti, Habermas e altri non negano gli effetti di lunga durata della secolarizzazione, riconoscendo che si tratta di un processo sociale che ha prodotto effetti rilevanti non reversibili; al tempo stesso, le religioni hanno ripreso un ruolo pubblico indubbio che gli Stati moderni non possono ignorare, attestandosi dietro la trincea della laicità assoluta. C'è in questa teoria, dunque, l'assunto di una relativa autonomia della forma-stato dalla religione e, al tempo stesso, del consolidarsi di un fenomeno che ha che fare con la secolarizzazione: si continua a credere negli dei, ma nessuno può pretendere di vincere e prevaricare sugli altri. Si crede nel relativo, di fatto, anche se qualcuno, proprio per questo, si indigna e rivendica con forza (sino all'agire violento) che c'è un solo dio e non altri.

Manca, a mio parere, nel dibattito sin qui ricordato un elemento importante della quaestio: il rapporto fra le forme moderne di credere (e le metamorfosi del sacro) e le mutate condizioni in cui si configura la formazione economico-sociale di tipo capitalistico. ${ }^{18}$ In altri termini, la discussione spesso si è concentrata sul rinnovato ruolo delle religioni nello spazio pubblico e, di converso, sulla posizione assunta dagli stati più o meno laici o secolari in rapporto al nuovo protagonismo delle religioni. In molti casi di studio (dall'Iran alla Turchia, dalla Polonia alla Spagna, dallo Sri Lanka all'India, tanto per citare luoghi dove diverse forze religiose sono state e sono in grado di mobilitare energie sociali, economiche e politiche, contestando l'a-confessionalità o la debole confessionalità degli Stati moderni), il protagonismo delle religioni finisce per essere un modo per parlare di politica quando le parole della politica non parlano più. I movimenti di tipo fondamentalista, ad esempio, spesso appaiono come attori religiosi che colmano un vuoto lasciato da partiti politici tradizionali e dalle grandi narrazioni ideologiche dell'Ottocento-Novecento. Ma in che relazione stanno sia le religioni forti (i movimenti di tipo fondamentalista) sia le forme del moderno credere nel relativo, individualizzate e orientate verso forme di spiritualità post-tradizionali, da un lato, e lo spirito del capitalismo neoliberista, dall'altro? La domanda, retoricamente, potrebbe essere formulata anche cosi: che ne facciamo dei nostri classici (della sociologia o della filosofia) quando cerchiamo di interpretare le forme post-secolari del religioso contemporaneo?

\section{La machina nel pensiero dei classici}

Sempre per rimanere fedeli all'immagine del deus ex machina, desidero richiamare, a questo punto, l'attenzione su un interessante risvolto narrativo che fra la fine dell'Ottocento e gli inizi Novecento, la sociologia assume, quando cerca di analizzare e raccontare la formazione di quella gigantesca e potente macchina sociale che è il capitalismo allo stato nascente. Siamo alle origini stesse della sociologia, quella che in modo convenzionale sarà chiamata la sociologia dei classici, cioè di tutti quegli autori che hanno costruito le prime teorie sul sociale.

Non ho lo spazio per passare in rassegna i molti autori che hanno immaginato e analizzato il primo capitalismo: ovviamente da Karl Marx con il suo Capitale ${ }^{19}$ (1867 primo libro, e poi postumi gli altri: 1885, 1894, 1905-10) sino a Georg Simmel che nel 1900 scrive un saggio intitolato Filosofia del denaro ${ }^{20}$ e, tre anni più tardi, un altro più

\footnotetext{
18 Sul tema, oltre alle riflessioni divenute ormai classiche di Z. Bauman, Modernità liquida, Roma-Bari, Laterza, 2011, si vedano le recenti ricerche condotte in T. Martikainen \& F. Gauthier (a cura di), Religion in the Neoliberal Age, London, Routledge, 2013 e F. Gauthier \& T. Martikainen (a cura di), Religion in Consumer Society, London, Routledge, 2013.

${ }^{19}$ Fra le molte edizioni si veda la recente versione Torino, UTET, 2017.

20 G. Simmel, Philosophie des Geldes, München-Leipzig, Duncker \& Humblot, 1900 (trad. it. Filosofia del denaro, a cura di A. Cavalli, Milano, Ledizioni, 2019).
} 
breve, quasi letterario, sulle metropoli e la vita dello spirito. ${ }^{21}$ Si potrebbe idealmente pensare di comporre una sorta di ipertesto ricavato da citazioni di questi autori così come di altri. Mi limiterò a citare solo due passi tratti rispettivamente, il primo, dall'Etica protestante e lo spirito del capitalismo di Max Weber ${ }^{22}$ (1904-1905) e il secondo dal Capitalismo come religione ${ }^{23}(1921)$ di Walter Benjamin. Vale la pena confrontarli, tenendoli sott'occhi entrambi, per poi passare ad un breve commento.

\section{Max Weber}

\section{Walter Benjamin}

Uno degli elementi costitutivi dello spirito del capitalismo moderno, e non solo di questo, ma della civiltà moderna (l'esistenza razionale condotta sulla base dell'idea del Beruf) è nato dallo spirito dell'ascesi cristiana.

Quando l'ascesi passò dalle celle dei conventi alla vita professionale e cominciò a ispirare l'etica intra-mondana, contribuì per parte sua a edificare quel possente mondo dell'economia moderna, legato ai presupposti tecnici ed economici della produzione meccanica, che oggi determina, con una forza coattiva invincibile, lo stile di vita di tutti gli individui che sono nati entro questo grande ingranaggio.

Doveva essere solo un leggero mantello, secondo la teologia puritana di Baxter, che doveva avvolgere le spalle dei santi in cammino verso la Terra promessa. Ma il destino ha voluto che il mantello si trasformasse in una gabbia di durissimo acciaio.

Quanto più l'ascesi imprendeva a trasformare il mondo a influire sul mondo, i beni esteriori di questo mondo acquistavano un potere sugli uomini crescente e, infine, ineluttabile.

Oggi il suo spirito è fuggito da questa gabbia e il capitalismo vittorioso non ha più bisogno di questo sostegno. Invero per gli ultimi uomini dello sviluppo di questa civiltà potrebbero diventare vere le parole specialisti senza spirito e gaudenti senza cuore $^{24}$
Il capitalismo è una religione perché serve essenzialmente all'appagamento delle stesse preoccupazioni, tormenti, inquietudini a cui in passato davano risposta le cosiddette religioni; essa è una religione puramente cultuale, la più estrema forse che mai si sia data; tutto, in esso, ha significato soltanto in rapporto immediato con il culto; non conosce nessuna particolare dogmatica, nessuna teologia. L'utilitarismo acquisisce, da questo punto di vista, la sua coloritura religiosa. Da qui la durata permanente del culto celebrato sans rêve et sans merci; non ci sono giorni feriali; non c'è giorno che non sia festivo, nel senso spaventoso del dispiegamento di ogni pompa sacrale, dello sforzo estremo del venerante. Questo culto è, al contempo, colpevolizzante e indebitante (verschuldend). Il capitalismo è presumibilmente il primo caso di un culto che non consente espiazione, bensi produce colpa e debito. Ricorre al culto non per espiare in esso questa colpa, bensi per renderla universale, per conficcarla nella coscienza e, infine e soprattutto, per coinvolgere in questa colpa il dio stesso e alla fine rendere lui stesso interessato all'espiazione. L'estensione della disperazione a stato religioso del mondo è ciò da cui si attende la salvezza. La trascendenza di Dio è caduta. Ma egli non è morto, è incluso nel destino umano.

Il capitalismo si è sviluppato in Occidente, (come aveva dimostrato Max Weber) in modo parassitario sul cristianesimo, in modo tale che, alla fine, la storia di quest'ultimo è essenzialmente quella del suo parassita, il capitalismo ${ }^{25}$

\footnotetext{
${ }^{21} \mathrm{G}$. Simmel, Le metropoli e la vita dello spirito, Roma, Armando, 1995.

22 M. Weber, Etica protestante e lo spirito del capitalismo, Milano, Rizzoli, 1991.

23 W. Benjamin, Il capitalismo come religione, Parma, Il Nuovo Melangolo, 2013 (con testo tedesco a fronte).

${ }^{24}$ Weber, Etica protestante e spirito del capitalismo, cit., p. 241.

${ }^{25}$ Benjamin, Il capitalismo come religione, cit., p. 12.
} 
Il capitalismo-macchina, soprattutto nella sua fase finanziaria, ha bisogno ancora di religione, se diventa esso stesso una forma collettiva di credere, con tanto di riti e culti? Sia le teorie (nate, non a caso, in ambiente statunitense) della scelta razionale o dell'economia religiosa (i beni di salvezza si vendono e si comprano al mercato delle fedi secondo le leggi della domanda e dell'offerta), sia gli studi sui processi di mercificazione di prodotti che incorporano simboli identitari di tipo religioso (merci che diventano perciò brand, oggetti di cult, per cui acquistandoli una persona si sente più pia o a posto con il proprio dio) sia, infine, l'emergere di ciò che per ora sono chiamate le mega-churches, ${ }^{26}$ grandi contenitori di rituali di massa in spazi architettonicamente distanti dai canoni sacri classici e moderni, dove abili imprenditori del carisma (attivi a livello transnazionale, grazie anche ai nuovi media in quel grande campo che include il pentecostalismo moderno, il neo-pentecostalismo e l'emergenza di organizzazioni cristiane non denominazionali) ${ }^{27}$ predicano il vangelo della prosperità e del successo economico via carismi e miracoli, sembrano tutte comprovare le previsioni che profeticamente sia Weber che Benjamin avevano intuito e descritto, sulla scia dell'opera di Marx, in una forma narrativa che, Michel de Certeau $^{28}$ avrebbe definito letteraria.

\section{Credere nel relativo e religioni forti}

Nella macchina del capitalismo neoliberista il lavoro è un costo e, se la persona che lavora non serve più, diventa uno scarto ${ }^{29}$ della società. Ritenere che questo sia un sacrificio necessario da accettare, sull'altare del futuro progresso che il capitalismo continua a promettere, non è altro che la metamorfosi dello spirito del capitalismo così come descritto da Weber e Benjamin. Si potrebbe anche parlare di capitalismo post-secolare, mettendo assieme l'intuizione weberiana della "gabbia d'acciaio" con quella più netta di Benjamin sulla capacità del capitalismo di diventare religione e culto quotidiano. Infatti, il post-secolare del capitalismo contemporaneo consiste nel fatto che esso non ha più bisogno del "sostegno di una religione", ma solo di vivere parassitariamente sulle religioni, sfruttando, a volte, il loro, anche furioso, vitalismo. Le forme di tale vitalismo possono essere descritte ricorrendo a delle figure tipiche, che fungono nel nostro caso da espediente letterario per contenere, in poche righe, quanto di complesso accade sotto le sacre volte della religione.

Danièle Hervieu-Léger ${ }^{30}$ ne ha felicemente tratteggiate due: quella del pellegrino e quella del convertito. Due figure che descrivono una religione in movimento. Si crede: più per scelta che per appartenenza fissata da una tradizione. L'individualizzazione del credere implica l'indebolimento di una religione determinata, che non riesce più a farsi cultura, semantica del vivere e del pensare di grandi aggregati umani e sociali. Allo stesso tempo, la mobilità nelle scelte di credere e non credere e, soprattutto, di credere a modo proprio, sciolgono i legami di appartenenza a una comunità credente, con una sede fisica, con il suo ambiente sociale e il suo territorio. Un credere de-culturalizzato ${ }^{31}$ e de-territorializzato, insomma. Gli habitus del cuore ricevuti (si nasce in una religione ancora oggi, anche se magari in famiglia non si ricevono più i primi generi di conforto spirituali e i primi insegnamenti religiosi) nell'ambiente sociale in cui si è chiamati a crescere e vivere, non sono più un'assicurazione sulla vita religiosa degli individui e sul destino sociale

\footnotetext{
${ }^{26}$ E. Pace, Cristianesimo extra-large, Bologna, EDB, 2018.

27 E. Pace \& A. Butticci, Le religioni pentecostali, Roma, Carocci, 2010.

28 'Le roman psychanalytique', cit., p. 125.

29 Questa parola è entrata a far parte del lessico teologico di Papa Francesco. Si veda quanto scrive nell'enciclica del 24 maggio 2015, Laudato Si'.

${ }^{30}$ Le pèlerin et le converti, Paris, Flammarion, 1999 (trad. it. Bologna, il Mulino, 1999).

31 O. Roy, La santa ignoranza, Milano, Feltrinelli, 2009.
} 
delle religioni. Ci si mette in movimento, in ricerca, spinti da scelte soggettive perché la religione della tradizione non convince più in tutto e in parte. Da qui la persistenza delle figure del bricolage o del 'butinage', ${ }^{32}$ del passare di fiore in fiore come l'ape per cogliere o consumare ciò che attrae di più. Si sceglie di credere e ci si muove nello spazio sociale (dilatato oggi dai nuovi media, che propongono nuove online religions) ${ }^{33}$ popolato da offerte religiose o di tecnologie spirituali le più varie e disparate. Ci si può arrestare anche per un certo tempo in un luogo preciso, accettando il processo di conversione e una nuova disciplina interiore, e/o del corpo. Tuttavia, tutto ciò può mutare, spingendo a passare da una chiesa a un'altra, da un gruppo spirituale a un altro, senza una reale fissa dimora. In altri termini, il convertito è un credente postsecolare, che può combinare stili di vita perfettamente moderni con la ricerca di modi per coltivare lo spirito. Non rifiuta la religione come se fosse un retaggio arcaico, la sente, invece, come un genere di conforto spirituale o di benessere mentale che può coerentemente convivere con il sentirsi moderni. Può anche sentire che la religione può essere una valida arma di critica agli ordinamenti dominanti in una società, arrivando anche a concepirla come la critica delle armi, unica alternativa possibile a un ordine del mondo ormai corrotto e assatanato.

All'interno delle due figure del pellegrino e del convertito troviamo tutti quei profili di nuovi credenti: di chi sente di essere rinato (born-again) alla fede; di chi concretamente riscopre antichi cammini o vie di pellegrinaggio e si mette alla prova, sia fisica che mentale, senza che tutto ciò si traduca in un ritorno "alla parrocchia" (in senso metaforico: alla vita impegnata nella religione di nascita); di chi, in entrambi i casi, riprende gusto per il sacro, ma non si spinge sino al punto di farne il motivo centrale della propria vita. La religione o le pratiche di tipo religioso possono continuare ad avere senso ma solo in una provincia di significato ben delimitata, che non necessariamente ha influenza su altre sfere della vita.

Le forme post-secolari del credere non sembrano in grado di contrastare il principio stesso della secolarizzazione moderna, la differenziazione delle sfere della vita sociale, ciascuna delle quali finisce per ubbidire a proprie regole interne di funzionamento, non facilmente permeabili dalle etiche d'ispirazione religiosa. Politica, diritto, scienza, economia ed eros seguono ciascuna una propria ragione che non può venir condizionata dalle religioni. Ecco perché post-secolare non significa ritorno del sacro o fine della secolarizzazione. Siamo andati oltre a quanto avevamo visto e vissuto nelle varie fasi del processo di secolarizzazione nel respiro lungo della storia moderna, soprattutto dell'Occidente storicamente plasmato dal cristianesimo. Per le religioni cristiane ma anche per quella ebraica, la modernità - almeno nel Vecchio Continente - ha voluto dire riconoscere all'individuo la libertà di credere e non credere, o di credere in forme diverse da quelle ufficialmente riconosciute da una Chiesa o da una Tradizione. Qualche Chiesa (quelle soprattutto uscite dalla Riforma) è arrivata prima di altre; le comunità ebraiche, invece, hanno conosciuto un'interna differenziazione nelle scelte di credere quando finalmente nell'Ottocento sono uscite dai ghetti sociali e culturali in cui per secoli erano state segregate. Il credere individualizzato, così come lo conosciamo dal secondo dopoguerra in poi, con tempi sfasati da Paese a Paese, secondo linee di frattura storiche fra Paesi di tradizione protestante, da un lato, e Paesi di tradizioni, rispettivamente, cattolica e ortodossa, dall'altro, è, a ben guardare, il frutto maturo dell'affermazione della libertà di credere; al contempo, dell'autonomia delle sfere della vita sociale rispetto a quella religiosa.

32 Un'espressione preferita oggi da un gruppo di studiosi soprattutto latino-americani. Si veda per tutti Y. Droz, E. Soares \& A.P. Oro, Le butinage religieux: regard anthropologique sur les pratiques religieuses, Paris, Karthala, 2014.

${ }^{33}$ E. Pace, La comunicazione invisibile: religioni e internet, Milano, San Paolo Edizioni, 2013. 
Il contemporaneo credere individualizzato non comporta, tuttavia, la fine della religione. Cambiano i modi di andare a cercare risposte di senso al vivere con maggiori gradi di libertà, cercandole, appunto, anche altrove, rispetto alle varie religioni di nascita. Mettendosi in movimento per tale ricerca, una quota sempre più consistente ${ }^{34}$ di donne e uomini del Vecchio Continente, può peregrinare senza fissa dimora, può restare ai bordi della religione di nascita, può cambiare fede religiosa senza che tale decisione suoni, in realtà, come definitiva.

Nei processi di cambiamento o di conversione, per ricorrere a una parola antica, troviamo una terza figura della mobilità religiosa contemporanea. Possiamo chiamarla il combattente. Vista da vicino questa figura rappresenta in genere una contraddizione interna a complessi sistemi di credenza religiosa. In un primo elenco esemplificativo, potremmo includere l'ebreo rigidamente osservante in fervente attesa del Messia, momento che vede ormai prossimo perché le frontiere territoriali dello Stato d'Israele stanno per coincidere con i confini biblici di Eretz Israel (la Terra Promessa). ${ }^{35}$ Allo stesso modo, il profilo di un evangelicale ossessionato dall'imminente fine dei tempi che diventa, soprattutto se un born-again, intransigente verso quanti, affiliati a Chiese del protestantesimo liberal, appoggiano le battaglie per i diritti civili delle persone di orientamento omosessuale o ritengono ammissibile entro certi limiti l'aborto. Oggi in India, a fronte di credenti che praticano pacificamente le diverse vie spirituali dell'induismo, si ergono furiosamente i militanti di movimenti che rivendicano l'hindutva, ${ }^{36}$ la difesa di un'identità nazionale e religiosa prendendosela con le comunità musulmane o cristiane, oppure lottando per ricondurre all'ovile quanti, appartenendo agli strati più bassi del sistema delle caste o dei varna, si convertono al buddismo, al cristianesimo o all'islam.

Anche in alcune società del Sud-est asiatico a maggioranza buddista (Sri Lanka, Myanmar, Thailandia) emergono fiere figure di monaci buddisti combattenti, per la difesa della purezza dei fondamenti e delle virtù religiose di un popolo, contaminato, a loro detta, da presenze straniere e perciò estranee all'identità di quel popolo. In tali casi siamo di fronte a ideologie nazionaliste etnico-religiose che alimentano movimenti di protesta, a volte aggressivi e violenti. Infine, in questa lista va inserita una figura che ha avuto e continua ad avere un impatto drammatico sulla storia contemporanea a livello mondiale. Alludo al jihadista, un born-again che dell'interpretazione fanatica dell'islam e del mito della comunità primitiva dei puri e santi seguaci di Muhammad fa la sua bandiera, per giustificare la teoria e la prassi della lotta armata per la restaurazione dello Stato califfale.

Le tre figure del pellegrino, del convertito e del combattente sono indici del cambiamento dei modi di credere, delle differenze che sussistono tra le forme dell'appartenenza e nel declinare il rapporto fra pubblico e privato. A ben guardare, paradossalmente, si tratta di forme relative di credere, nel senso che esprimono una differenziazione interna alle singole religioni che nessuna autorità religiosa sembra in grado di ricondurre a ragionevole unità, o uniformità di comportamenti. Il fondamentalista-combattente, per distinguerlo dal fondamentalista-paziente che non pensa di imbracciare il mitra o farsi saltare con una bomba alla cintura, rappresenta il punto estremo del paradosso del moderno credere nel relativo. Apparentemente, egli

\footnotetext{
${ }^{34}$ La ricerca longitudinale sui valori degli europei da venti anni attesta che tale quota si aggira su un terzo della popolazione: vedi L. Halman, I. Sieben \& M. van Zundert, Atlas of European Values: Trends and Traditions at the Turn of the Century. Leiden, Brill, 2011.

${ }^{35}$ R. Guolo, Terra e redenzione, Milano, Guerini e Associati, 1997 e E. Pace, 'The Messianic Movements and the Sacralization of the Territory', in: REVER/Revista de Estudos da Religião, 3 (2019), pp. 2-16.

${ }^{36}$ G. Battaglia, L'altro fondamentalismo, Napoli, Guida, 2015 e C. Jaffrelot, Les nationalistes hindous, Paris, Presses des Sciences PO, 1993 e, dello stesso autore, 'India: The Politics of (Re)Conversion to Hinduism of Christian Aboriginals', in: P. Michel \& E. Pace (eds.), Religion and Politics, Annual Review of the Sociology of Religion, Leiden, Brill, 2011, pp. 195-215.
} 
è convinto che Dio sia dalla sua parte, di possedere la verità e di incarnare il modello del puro credente, perciò, come scriveva ironicamente Amos $\mathrm{Oz}$, 'ti ama in nome della verità che ama e per questo ti uccide: lo fa per il tuo bene'. ${ }^{37}$ Questi si trova costretto, tuttavia, a rappresentarsi i propri correligionari che non seguono la sua strada come dei nemici, alla stessa stregua di quanti appartengono ad altre fedi ritenute avversarie (nel linguaggio dei combattenti dei gruppi armati di matrice ideologica musulmana, ad esempio, sono evocati come i crociati e i sionisti). Crede nell'assolutezza della sua fede, ma si trova a doversi battere contro fratelli e sorelle che, per nascita e cultura, condividono la sua stessa fede.

Nel romanzo Il fondamentalista riluttante, ${ }^{38}$ l'autore Mohsin Hamid descrive il processo di trasformazione di un brillante analista finanziario di Wall Street in un militante fondamentalista. La frase che l'autore mette in bocca al protagonista, Changez Khan, è significativa: 'Vidi crollare prima una e poi l'altra delle torri gemelle del World Trade Center. E allora sorrisi'. Il sorriso anticipa il lento cambiamento che avviene in lui, che lo porta a tornare in Pakistan e ad avvicinarsi ai movimenti salafiti (integralisti), a convertirsi agli stili di vita propri di un militante islamista, spogliandosi degli abiti e degli habitus mentali propri di un individuo moderno, ben integrato nella società americana, senza più forti radici culturali con il Paese di origine dei suoi genitori né appassionato più di tanto alla religione.

C'è un luogo dove la polarizzazione fra forme del credere nel relativo e i profili delle religioni forti (espressi dai movimenti e gruppi di tipo fondamentalista nelle principali grandi religioni mondiali) è messa in scena in modo evidente, nel senso letterale del termine. Questo luogo è il mondo della cybereligion. ${ }^{39} \mathrm{Chi}$ naviga in rete, può trovare sia siti aperti all'invenzione di nuovi repertori religiosi da parte di chiese, sette, monasteri, gruppi mistici, guru e maestri spirituali, sia l'estensione online delle dottrine e delle pratiche ufficiali di questa o quella istituzione o autorità religiosa. Può anche, infine, imbattersi in gruppi di tipo fondamentalista, che si fanno interpreti della verità assoluta di cui si sentono portatori e strenui difensori. Se ci mettiamo, per un momento, nei panni di un navigatore della rete, magari appartenente alla coorte di quei nativi digitali indifferenti o lontani dalle rispettive religioni di nascita, l'impressione che ricaveremmo da quanto circola liberamente nel web può essere di due tipi. 0 di un mondo in cui la varietà e la differenziazione sono cosi manifeste da rafforzare la convinzione che credere sia una scelta ormai soggettiva e che non esista un'autorità capace di garantire la verità assoluta. Oppure che in quel mondo c'è chi offre una prospettiva forte, di certezze assolute; il passo successivo è di andare a cercare i gruppi che nella realtà possano soddisfare tale aspettativa. In ogni caso, anche questa scelta sarà, una volta ancora, soggettiva.

\section{Inattese affinità elettive}

Quali sono le affinità elettive fra le forme del credere nel relativo e la macchina del capitalismo neoliberista? La domanda, che riecheggia l'argomentazione principale della tesi di Weber sullo spirito del primo capitalismo "calvinista", può essere formulata anche altrimenti. Nelle moderne forme del credere, caratterizzate da gradi di libertà (la scelta individuale) molto più ampi rispetto a quelli concessi a chi si trova sotto il controllo sociale che le religioni hanno esercitato per generazioni e generazioni su interi popoli, la scelta di credere si dispone lungo un ideale asse con due estremi.

\footnotetext{
37 Contro il fanatismo, Milano, Feltrinelli, 2004, p. 18.

38 Torino, Einaudi, 2008. Da questo romanzo è stato tratto l'omonimo film del regista Mira Nair nel 2012.

39 La letteratura sul fenomeno è ormai diventata cospicua, grazie all'accumularsi di ricerche e riflessioni teoriche negli ultimi venti anni. Per un bilancio rinvio a E. Pace \& G. Giordan, 'Digital Altars', in: Historia Religionum, 2010, 3, pp. 77-94; H. Campbell, Digital Religion, New York, Routledge, 2012; F. Vecoli, La religione ai tempi del web, Roma-Bari, Laterza, 2013.
} 
Lo si può percorrere andando dall'intensità di una ricerca di senso, che può essere soddisfatta in vari modi ivi compresa la scelta di porsi sotto la tutela di un maestro o di una guida spirituale, fino al consumo di beni simbolici, magari incorporati in un oggetto-merce da comprare e da indossare sul proprio corpo. Tra questi due estremi, possiamo poi includere una gamma di scelte che si collocano in una sorta di terra di mezzo, ${ }^{40}$ fra credenza a bassa intensità religiosa e non credenza, fra indifferenza e curiosità momentanea nei confronti di segni e simboli religiosi. In tutti questi casi, l'individualizzazione del credere può mettere in crisi chiese storiche o tradizioni consolidate, favorendo, fra i vari effetti sociali inattesi, l'affinità del bisogno di religione con lo spirito del mercato, capace di trasformare un simbolo religioso in un brand. Ciò può dare ragione della spinta delle nuove formazioni religiose ad agire sul mercato, adottando strategie commerciali nella comunicazione dei loro messaggi, per competere con altri attori che si muovono allo stesso modo.

Può accadere, allora, che la produzione dei beni simbolico-religiosi che circolano in un mercato globale non abbia più confini culturali precisi. Siamo oltre Weber, in tali casi, e più vicini al pensiero di Benjamin. Detto in altri termini, laddove prevale uno sviluppo economico dominato dal capitalismo neoliberista può accadere - non è detto che lo faccia sempre e che sia un destino storico irreversibile - che le religioni più affini a tale modello siano quelle che predicano la prosperità economica, se si è credenti e fedeli convinti. Avere successo nella vita, arricchendosi e migliorando il proprio status, diventa allora una prospettiva etica proposta da nuovi predicatori, leader carismatici e maestri digitali, del tutto coerente con le regole del gioco di una formazione socioeconomica che ha bisogno funzionalmente di individui che sappiano calcolare i costi e i benefici anche nel caso delle loro scelte religiose. ${ }^{41}$

In Cina, da quando le autorità del partito comunista al potere, negli anni Novanta, hanno assecondato lo sviluppo di un capitalismo rosso, accettando la logica del mercato, competendo a livello mondiale, le religioni sono tornate di moda. ${ }^{42}$ II regime ha recuperato il confucianesimo, il taoismo e il buddismo, sostenendo che esse rappresentano l'anima culturale del popolo cinese. I valori morali da esse promossi, sono considerati dalla classe dirigente un patrimonio culturale irrinunciabile, perché utile a sostenere il grande sforzo economico che la Cina sta compiendo. Da qui la relativa liberalizzazione, anche se occhiuta e guardinga, delle altre fedi, come il cristianesimo e l'islam. ${ }^{43}$ Rispetto al cristianesimo, in particolare, è interessante richiamare l'attenzione sullo sguardo tollerante che il regime riserva per le cosi dette chiese domestiche, sovente create e finanziate da piccoli e medi imprenditori privati che gravitano nell'area del nuovo cristianesimo di tipo evangelico o pentecostale. Una religione privatizzata, atomizzata e senza mega-organizzazioni sovranazionali non disturba il potere costituito, che vede in essa un genere di conforto spirituale ed etico, adatto a individui che devono dare tutto per sostenere la produttività del capitale e vincere la competizione globale.

L'esempio cinese, paradossalmente, è una buona prova empirica dell'esistenza delle religioni post-secolari. Uno stato autoritario avverte che lo sviluppo economico di tipo capitalistico, aperto al mercato e alla competizione finanziaria, diventa insostenibile senza l'ausilio della religione e del relativo sistema di norme, capaci di

\footnotetext{
40 Secondo l'espressione usata da A. Castegnaro, C'è campo?, Venezia, Marcianum Press, 2010 per descrivere gli atteggiamenti delle nuove generazioni nei confronti del cattolicesimo in Italia.

41 Su questo tema rinvio a J. Garcia-Ruiz \& P. Michel, Et Dieu sous-traita le salut au marché, Paris, Armand Colin, 2012 e P. Michel, A. Possamai \& B.S. Turner (eds.), Religions, Nations and Transnationalism in Multiple Modernities, London, Palgrave MacMillan, 2017.

${ }^{42}$ M. Scarpari, Ritorno a Confucio. La Cina di oggi fra tradizione e mercato, Bologna, il Mulino, 2015.

${ }^{43} \mathrm{~F}$. Yang, Religion in China, Oxford, Oxford University Press, 2011 e dello stesso autore, Atlas of Religion in China, Leiden, Brill, 2018.
} 
disciplinare menti e corpi di individui lasciati soli dalle grandi tradizioni del credere. Queste ultime, nel caso cinese, sono state scientificamente distrutte dal maoismo e dalla rivoluzione culturale. Dunque, non risorgono oggi come per incanto. Sono rianimate dal basso, inventando modalità di credere compatibili con le regole del gioco politico e di quello del mercato, oppure sono imposte come quadri sociali della memoria collettiva dal regime stesso, preoccupato che l'individualismo senza più padri né maestri laici e rivoluzionari corroda il sistema di potere su cui si regge incontrastato il partito comunista. È proprio pensando a questo stato delle cose che ho scelto il deus ex machina come immagine di apertura di questo articolo.

Diventato una macchina mondiale, senza più rivali e alternative dopo la caduta del Muro di Berlino, il capitalismo neoliberista ha bisogno di un consumatore ben integrato e non di apocalittici disintegrati. Un consumatore convinto di poter scegliere liberamente tra le numerose merci che gli vengono incontro in ogni momento della sua vita, che non gli danno tregua non solo quando accede ai vecchi media, ma anche quando si connette ai nuovi; quando vuole prenotare un volo online o fare una transazione commerciale. Sta acquistando in questi casi un prodotto particolare, ma è sollecitato da messaggi che gli illustrano le virtù e le meraviglie di altrettante merci. Possono pure chiudere la domenica i centri commerciali, come da varie parti si chiede in nome del riposo settimanale tradizionalmente consacrato al Signore, ma grazie a internet tutti i negozi virtuali sono aperti e accessibili: posso comprare, e farlo navigando in un mare di pubblicità, sans rêve et sans merci, come dice Benjamin. La tiratura dei giornali continua a scendere. Posso leggere online notizie e articoli di fondo di tutte le testate che voglio consultare. Mentre lo faccio, i miei occhi e la mia attenzione sono costretti a scansare, come tanti ostacoli alla lettura, messaggi pubblicitari che finiscono per omologare le notizie alle merci, entrambe lanciate dalla pagina virtuale dei giornali. Chi produce ha interesse a proporre modelli di consumo che continuano a essere, a loro volta, produttori di stili di vita. Attorno a oggetti, a volte di valore merceologico non elevato, si creano comunità di consumatori che fanno di quel particolare prodotto un culto (cult). Grazie al web, infatti, la formazione di comunità di consumatori fidelizzati è diventata più facile. Attraverso il consumo si cominciano a condividere un linguaggio, comportamenti, rituali e aspettative. ${ }^{44}$ Se lo stato moderno aveva secolarizzato i concetti della teologia cristiana, ${ }^{45}$ il consumismo tende oggi sempre più a secolarizzare segni e simboli religiosi. Le merci possono incorporarli e, in tal modo, una religione può anche diventare un brand. Un esempio, fra i tanti che scelgo però proprio perché contro-intuitivo, ci proviene da una realtà come l'islam, che secondo gli stereotipi più comuni in Occidente rappresenta quanto di più arcaico e antimoderno che ci sia. Ebbene, l'islam è diventato anche un brand, un marchio che compete nel mercato mondiale. ${ }^{46}$

Nel 2019, secondo la rivista Fortune, nei Paesi a maggioranza musulmana sono stati spesi 484 di dollari in vestiti e scarpe, più di Giappone e Italia messi assieme, e più del mercato dell'abbigliamento complessivo dell'UK, Germania e India. Nell'ottobre 2016, Dubai ha ospitato per la prima volta la Fashion Week, con la presenza dei più famosi designer europei e del Medio Oriente (da Chanel a Stella McCartney); l'anno dopo, Dolce\&Gabbana annunciava l'apertura di una boutique per bambini nel Dubai's Mall of the Emirates. Gli e-tailers Moda Operandi e Net-A-Porter offrono, da alcuni anni, una speciale linea di vestiti per il mese di Ramadhan (dedicato al sawm, al digiuno). In tutto il variegato e complesso mondo musulmano (dal Maghreb all'Indonesia e la Malaysia, passando per il Medio Oriente e i ricchi Paesi del Golfo), la

\footnotetext{
44 Sulla formazione di tali comunità si veda R.V. Kozinets, Netnograpohy, London, Sage, 2009.

45 Secondo la tesi del giurista, sociologo e politologo tedesco C. Schmitt, Politische Theologie, MünchenLeipzig, Duncker \& Humblot, 1922 (trad. it in Le categorie del politico, Bologna, il Mulino, 2012).

${ }^{46} \mathrm{~F}$. Tarzia (a cura di), Religioni nella metropoli, Roma, Il ManifestoLibri, 2018.
} 
rilevanza del fattore $\mathrm{R}$ (religione) negli orientamenti di consumo è notevole, soprattutto fra le nuove generazioni. Vestirsi, nutrirsi, fare affari e scegliere un partner sono diventati comportamenti sempre più codificati. Non tanto in base a precetti religiosi astratti, ma quanto piuttosto in base alla ricerca di stili di vita compatibili e coerenti con la shari'a. La conformità è vissuta (e, dunque, non più avvertita come imposta) attraverso il consumo del bello, del buono e del gusto. Che il gusto conti, lo prova l'espansione del mercato globale dello halal, che non riguarda solo la carne macellata secondo il rituale sharaitico, ma anche tutti i prodotti certificati senza derivati dal maiale o senza tracce di alcol (compresi i profumi e i cosmetici, di cui si fa un ampio consumo). Questo mercato, il cui fatturato ha toccato nel 2017 la ragguardevole cifra di un trilione e mezzo di dollari (si calcola che arriverà a 2,6 trilioni nel 2023), ${ }^{47}$ copre tutto il ciclo merceologico: dalla produzione alla certificazione di qualità (religiosa), dalla preparazione allo stoccaggio delle merci. La Malaysia è diventata, negli ultimi venti anni, il maggiore centro di produzione e certificazione halal nel mercato globale per i musulmani. ${ }^{48}$ Nel 2002, l'imprenditore franco-tunisino, Tawfiq Mathlouthi lanciava la Mecca-Cola, a imitazione della bevanda Zam-Zam prodotta sin dal 1980 in Iran. La Mecca-Cola oggi è venduta in 60 Paesi nel mondo, per una media di 20 milioni di litri all'anno, distribuita non dalle grandi catene commerciali, ma solo attraverso le reti di piccoli negozi sparsi fra l'Europa, gli Stati Uniti e il Canada. Da qualche anno è iniziata una campagna di diffusione in alcuni Paesi dell'Africa sub-sahariana. Una percentuale (il 2\%) dei profitti è devoluta a una fondazione per gli aiuti alla popolazione palestinese nei territori occupati.

Quanto sin qui detto mostra come l'islam sia entrato integralmente a far parte del mondo dei consumi: senza troppi problemi etici, dal momento che sotto l'apparenza del rispetto di tradizioni e precetti religiosi, le nuove generazioni, in particolare, affermano la loro modernità anche attraverso il consumo di simboli religiosi. Il mondo dei consumi riesce, infatti, a soddisfare la doppia esigenza del poter scegliere individualmente (ciò che è bello, buono e gustoso) e della possibilità d'incorporare la religione negli stili di vita. Si tengono assieme, in tal modo, desiderio soddisfatto e bisogno d'identità collettiva, via religione. Perciò, la strategia del marketing è al tempo stesso orientata a stimolare il consumo offrendo, simultaneamente ampie chances di scelta individuale ${ }^{49}$ che possano apparire conformi ai principi e ai precetti della legge coranica.

\footnotetext{
47 Global Halal Market/Statistics \& Facts, New York, Statista Inc., 2018, www.statista.com/topics/ 4428/global-halal-market (14 dicembre 2020).

${ }^{48}$ Sul tema F. Bergeaud-Blackler \& J. Fisher (eds. ), Halal Matters, London, Routledge, 2015 e F. BergeaudBlackler, Le marché halal ou l'invention d'une tradition, Paris, Seuil, 2017.

49 Lo dichiara con molta chiarezza Rabia Z, una giovane Muslim Designer, che ha creato una linea di moda chiamata Conservative Chic Fashion, in un'intervista rilasciata alla Televisione Al-Jazeera, il 1 gennaio 2016, in una trasmissione dedicata alle firme emergenti della moda islamica: 'Sono felice nel constatare che la moda straniera sia entrata nel mondo dell'industria della moda castigata. Ciò implica maggiori scelte per il consumatore. Tuttavia, desidero chiarire che la produzione di capi di abbigliamento venduti come brand non è solo finalizzata a far quattrini. Ci metto l'anima nel mio lavoro perché per me è un modo per rafforzare le donne musulmane dando loro la possibilità di scegliere, per potersi esprimere liberamente'. Per un'analisi più specifica di questo aspetto della moda femminile, si vedano N. Göle, 'The Voluntary Adoption of Islamic Stigma Symbols', in: Social Research, 3 (2003), pp. 809-828; M.N. Hashim, 'The Constructs Mediating Religions' Influence on Buyers and Consumers', in: Journal of Islamic Marketing, 2 (2010), pp. 124-135; H. Godazgar, 'Islam in the Globalized World: Consumerism and Environmental Ethics in Iran', in: L. Thomas (ed.), Religion, Consumerism, and Sustainability Paradise Lost?, Basington, Palgrave MacMillan, 2011; F. Mohsina et al., 'Shari'a Compliance in Building Identified Islamic Brands', European Journal of Business and Management, 5 (2013), pp. 10-16 (special issue on Islamic Management and Business); R. Lewis, Muslim Fashion: Contemporary Style Cultures, Durham, Duke University Press, 2015; F. Shirazi, Brand Islam: The Marketing and Commodification of Piety, Austin, University of Texas Press, 2016.
} 
L'etica musulmana, dunque, sembra ingranare con lo spirito del capitalismo neoliberista. In Indonesia, uno dei Paesi a stragrande maggioranza musulmana, ad esempio, l'interesse a consumare merci garantite come doc-musulmane, non costituisce un naturale rifugio da parte di chi si sente spaesato e indifeso di fronte al capitalismo globale. Si tratta piuttosto di un nuovo habitus mentale che si è affermato proprio in relazione dialettica con il turbo-capitalismo mondiale. Tale habitus è l'espressione di un'etica individualistica dell'auto-realizzazione, che si affida anche al consumo di merci, oggetti, cibi e vestiario che incorporano simboli religiosi. In tal modo, nei mondi vitali degli individui si configura una visione etico-religiosa compatibile con lo spirito del capitalismo neoliberista. Allo stesso modo tutto ciò, infine, stimola lo spirito d'impresa sia nel caso di imprenditori musulmani per nascita e formazione, sia nel caso in cui imprenditori non-musulmani creativamente inventino linee di merci e prodotti che possano apparire, agli occhi dei consumatori, compatibili con i loro precetti religiosi. ${ }^{50}$ Un solo esempio per tutti. Due giovani stiliste che lavorano e risiedono a Londra hanno creato la Sister Couture per una clientela femminile musulmana, una linea di capi di abbigliamento il cui obbiettivo è quello di combinare: 'faith, modesty, style \& inspiration: promote your faith, uphold your modesty, define your style and be an inspiration to others' ${ }^{51}$

\section{Conclusione}

Gli esempi sin qui brevemente illustrati e le riflessioni che li hanno preceduti confortano l'ipotesi che ho avanzato all'inizio dell'articolo. Le religioni cercano di far immaginare unito un mondo sociale che è diviso, differenziato, conflittuale, e che lo è innanzitutto in senso religioso: a essere molteplici, infatti, sono le stesse visioni religiose del mondo e della vita anche per quanto riguarda la visione religiosa del mondo e della vita. Il credere nel relativo si è imposto anche all'interno delle religioni storiche, prodotto maturo della secolarizzazione. Parafrasando Weber, la disciplina morale cui le religioni invitavano a sottomettere le anime e i corpi seguendo il modello ascetico, è uscita dalle 'celle monastiche' ed è diventata virtù del vivere nel mondo (intra-mondana). Essa, in tal modo, ha finito per essere sedotta dallo spirito del capitalismo tardo-moderno, che ha bisogno di individui che si sentano liberi di scegliere e di distinguersi ma che, allo stesso tempo, possano ritrovare tracce del sacro nel simulacro delle merci.

A chi ha nostalgia del senso di comunità e di appartenenza che le religioni si sforzavano di predicare, il capitalismo neoliberista offre proposte merceologiche che incorporano segni e simboli religiosi. La standardizzazione dei comportamenti individuali non appare più come un dovere imposto dall'esterno, da una autorità o da una consolidata tradizione religiosa, ma si presenta come una confortevole libera scelta di conformità alle regole del mercato. Conformarsi alle norme religiose non appare più e solo come l'esercizio di una virtù ascetica, ma anche come un piacere estetico che nasce dal desiderio di sentirsi moderni. Ecco perché il mondo dei consumi, che prende concreta e quotidiana forma negli stili di vita di individui e di gruppi di individui (anche vasti), non chiede di sacrificare il bisogno d'identità e di ricerca di senso religioso da dare alla propria vita. ${ }^{52}$ Chiede invece di orientare tale bisogno verso merci certificate, che garantiscano il rispetto delle regole religiose del caso

\footnotetext{
50 Su questi temi rinvio rispettivamente a D. Rudnycky, 'Spiritual Economics: Islam and Neoliberism in Contemporary Indonesia', in: Cultural Anthropology, 1 (2000), pp. 98-120; D. Assadi, 'Do Religion Influence Customer Behaviour?’, in: Cahiers du CEREN, 5 (2003), pp. 2-13; J. Grant, Made with: Brands, Creations and Entrepreneurs from Emerging Global Interland, London, LID, 2013.

51 Dalla homepage del sito http://www.urban.muslim.co.uk.

52 Un'interessante ricerca etnografica, a tal proposito, è stata condotta da P. O'Connor, Skateboarding and Religion, London, Palgrave Macmillan, 2020.
} 
(alimentari o di vestiario). In tal modo le virtù ascetiche trovano realizzazione e celebrazione in quelle della merce. Un processo di secolarizzazione che non rende insensate le moderne scelte di credere da parte degli individui. L'ascesi intra-mondana che il consumismo moderno rende possibile è un altro modo, fra i tanti possibili, di comprendere le metamorfosi del sacro via religione nel mondo contemporaneo.

\section{Parole chiave}

secolarizzazione, ritorno del sacro, post-secolare, capitalismo neoliberista, religione come brand

Enzo Pace è professore di sociologia delle religioni del Corso di Laurea Magistrale in Scienze delle religioni, Università di Padova e Ca' Foscari di Venezia, visiting professor all'EHESS (École des Hautes Études en Sciences Sociales, Parigi) ed è stato presidente dell'International Society for the Sociology of Religion (ISSR). Ė co-editore, assieme a L. Berzano e G. Giordan, dell'Annual Review of the Sociology of Religion. Recenti pubblicazioni: Le religioni nell'Italia che cambia (a cura di), Roma, Carocci, 2013; Religious Pluralism: Framing Religious Diversity in the Contemporary World (ed.), New York, Springer, 2014; The Changing Soul of Europe, London, Routledge, 2015; Sociologia delle religioni, Bologna, EDB, 2016; Cristianesimo extra-large, Bologna, $\mathrm{EDB}, 2018$.

Dipartimento di Filosofia, Sociologia, Pedagogia, Psicologia Applicata

Università di Padova

Sede di Sociologia: via Cesarotti 12

Padova, 35122 (Italia)

vincenzo.pace@unipd.it

\section{SUMMARY}

\section{Deus ex machina}

\section{Contemporary Forms of Believing in the Relative}

Secularization continues to produce its effects over the long term, despite the conspicuous recovery of public space regained from 1980 to today by the major world religions. The alleged return of the sacred or the alleged revenge of God, however, does not seem able to contrast the triumph of capitalism as a religion, to take up the formula used by Walter Benjamin. The modern consumer society saves the salvable, incorporating the sacred into the commodities, making the goods a sign of identification, a cult. The appearance of strong religions, so-called fundamentalisms, constitutes a specular and high response to the forms of believing in the relative in the societies of late secularization.

The author starts from the prophetic visions on the magnificent fortunes of capitalism, advanced by Max Weber and Walter Benjamin, analyzing the process of commodification of religious symbols in contemporary affluent societies. Therefore, there is not the return of God who was presumed dead. Rather the machine of neocapitalism saves God by making him prodigiously incarnate in the beauty of goods. 